\title{
Research on Intelligent Monitoring System Design based on Wireless Sensor Networks
}

\author{
LI Bin ${ }^{1, a}$ \\ ${ }^{1}$ Dean's office,Chongqing college of electronic engineering Chongqing 401331, China \\ axiaohuo111@sina.com
}

Keywords: Intelligent Monitoring System, Design, Wireless Sensor Networks

\begin{abstract}
Now China is ushering in a "digital city" "Digital Communities" Construction and "low-carbon economy" boom, 3G technology and Internet application development for smart home also play a very important role, and intelligent home can the effective realization of "low-carbon economy," "green economy", smart home system based on wireless sensor network, will effectively solve the many problems faced by ordinary wireless products.
\end{abstract}

\section{Introduction}

In the 21st century, with advances in science and technology level, quality of life of the people have become more sophisticated; it makes the concept of people's life has undergone tremendous changes. Nowadays, people have to pursue the family's living standards improved significantly, not just ordinary household equipment or increase physical space, but the security of family life, easy to have higher expectations, want to live in a more modern, safe and comfortable indoor environment. While scientific and technological progress to bring a variety of continuous upgrading of modern high-tech products, but also brings a lot of insecurity, such as in recent years due to the use of high-power electrical appliances: electric dig, electric oven caused by improper fire, explosion or events that occur frequently. Meanwhile burglary, theft and other criminal acts in the community caused by criminal elements but also to people's lives caused great distress. Thus, in the modern intelligence community and in the family, the family environment, real-time monitoring, can effectively prevent this from happening, or at least reduce the harm caused by the above situation. Therefore, the study of home security monitoring system is necessary, and it is worth investors to invest.

Traditional smart home systems generally use a wired manner established: such as LonWorks, CEBus, X-10, CANBus like. Wired networking technology as a traditional networking technology, the advantage is more mature, but there are also many deficiencies in the place: for example, wiring cumbersome, takes up a certain amount of interior space, cumbersome wiring affect the aesthetics of the room, and finite way wiring poor scalability. Compared to the limited networking, wireless networking advantages are obvious: no additional wiring, flexible configuration, easy to maintain, easy to remove and add network devices, and therefore more suitable for the application of intelligent home security monitoring aspect. Wireless networking is also includes a variety of wireless communication technologies: infrared communication technology, Win, Bluetooth, UWB (ultra-wideband technology), ZigBee, etc. In recent years, the rapid development of wireless communication technology in various fields and industry is widely used, intelligent home security monitoring system internal network using wireless communication technology is the trend.

\section{The Development Status and Application of Wireless Sensor Networks}

Wireless sensor network is considered the most important technology of the 21st century. US "Business Week" on the future of technology trends to predict, and that the wireless sensor network technology is very influential in the 21st century technology. At this stage, wireless sensor communication technology is in the development stage, its theoretical and practical applications of technology are to be further improved to achieve substantial, large-scale application is still a long way to the technology is a relatively new technology, ZigBee protocol is designed for wireless 
sensor network protocol developed with low power consumption, low cost, low power consumption, its transport, energy saving networks to maximize. Built with ZigBee wireless networking technology that can accommodate much 65536 nodes, and has high robustness, these features make ZigBee protocol more and more applications in wireless sensor networks. With the upgrading of information technology in recent years, more and more low-cost computers, wireless networks have been applied in the following areas:

The Home Control. Family in a variety of electrical appliances, lighting equipment, as well as data from various sensors preclude the collection of system components inside a home control network through a wireless device networking technology, which is a typical application in indoor scenario. This network, the data processing nodes can easily control center for each child node device monitoring and control, and can be through broadband or GPRS technology, allowing users at home can not achieve control over home devices.

Smart Metering. Smart metering (smart metering) is a smart grid (smart grid) key, is a kind of real-time power usage monitoring system, it is generally for large-scale electricity places, such as arm office buildings, residential areas and large mechanized enterprises. Compared to the traditional meter reading, smart metering have higher requirements, because it has more frequent meter reading times, the need for more accurate meter reading accuracy, so you can make the right treatment.

The Medical Care. Medical problems have been a great country to be a problem. Now, as the global aging trend has accelerated, and the demand for health care, more and more, society needs to invest more human and material resources to conduct medical monitoring care. In such a context, combined with wireless sensor networks of high-tech medical care was presented. Some hospitals by measuring body temperature, heart rate, blood pressure, body sensors worn on the patient, allowing the wireless network will gather in front of the information to the doctor, so that a doctor can monitor several patients, and make a timely response, greatly It reduces the human physical labor, and increase efficiency. Meanwhile, the health care system can be based on some common data analysis and processing conditions, the application applies not only in the hospital, in the family also has a good versatility.

Environmental Monitoring. In the process of industrialization, people at the expense of the environment, resources in exchange for the cost of environmental problems caused by the development of more and more serious, now, the world regarded as a priority for sustainable development, environmental issues have been of great importance. Than the traditional wired networks, wireless networks have a compact, easy wiring, low cost, combined with more sophisticated sensor technology, wireless networks can easily collect environmental data.

\section{The Key Technology Analysis of Wireless Sensor Networks}

Self-Organizing Network Technology. Self-organizing wireless network technology that is randomly laid sensor network multi-network integration technology. Ad-hoc network is a multi-hop temporary autonomous system consists of a set of (mobile) terminal nodes with wireless communication transceiver devices, each of which (mobile) terminal system has both routers and hosts two functions. First, as the host, the terminal needs to run user-oriented applications; the second is as a router, the terminal needs to run the appropriate routing protocol. The route between the network node usually consists of a multi-hop (Hop) composition, does not require network infrastructure, can be anywhere, build anywhere fast.

Distributed Processing Technology. System to achieve each sensor node as a network node at the same time, sensor data fusion, intelligent filtering and other processing; establish systems based on TCP / IP protocol stack architecture with Internet network. Traditional routing protocols need to be modified to adapt to the dynamic changes in the network topology needs. Based on self-organizing network protocol stack can be freely adapted to various types of network topologies custom number, transport layer adapted to the wireless network end to end reliable service.

Detection Algorithm Design. System detection algorithm design process is to complete a series of multi-sensor information fusion algorithm design process. The so-called information fusion is 
information from multiple sensors or sources integrated process to arrive at a more accurate and reliable conclusions. Like humans and other living things, they have a basic function, namely multi-sensor data fusion. After the human use of eyes, ears, nose, limbs and other organs function to detect scene, sound, touch, and smell, and humanity itself a priori knowledge synthesis, and to make certain judgments and estimates and their surrounding environment around the event. Since the human sense of the palace has different measurement characteristics, which can detect a variety of chemical and physical phenomena in different ranges, this process is adaptive, and complicated. All kinds of information it images, smells, sounds and describe the physical shape or the like into the environment on the basis of interpretation.

Image Transmission Technology. Channel processing is to ensure that the image information is transmitted in the channel, the received image information to achieve the required quality, free from outside interference, or cause interference within the allowable range. Source process is compressed transfer amount of image information, to accommodate the transfer rate of the transmission channel and bandwidth. Image transmission technology, there are two different ways to be divided into analog and digital transmissions.

\section{Intelligent Monitoring System Design based on Wireless Sensor Network}

First, the system initialization process, and detecting the corresponding hardware module is not connected well, including the main chip IO port configuration, configure ZigBee RF module, GPRS module initialization and so on. If the initialization successful start into the main program execution, wait for key interrupt event and the wireless module interrupt event occurs, and then enter the interrupt handler. Monitoring center security system after program initialization, interrupt event has been in the judgment, the event is determined mainly includes two aspects, one is to test the key, on the other hand is detected wireless communication interruption has occurred. The main button is used to set the parameters of the system to achieve human-computer interaction and wireless communication is used to trigger an interrupt ZigBee node to send data up.

Parameter real-time security monitoring system is adjustable can be for different users, or with a different user environment set different alarm thresholds, the formation of different alarm parameters. Parameters are in the main control center through the LCD keyboard display module for setting the parameters in which the master node is set directly from the keyboard and terminal node parameters wirelessly transmit it. Where the parameters of the master node is set up directly after save in Flash, and parameters of the terminal node after a good set up, also need to send separately by ZigBee RF module to module to each node. The main parameters of the master node are used to set the total number of mobile phone users and the number, as well as to determine how much child node module. The terminal node parameters is used to determine the presence or absence of certain terminal module functions, including infrared sensor, camera, CO sensors, smoke sensors. Because it involves wireless communications, it is necessary to transfer parameter data format control center to make a ZigBee node unified regulations.

FLASH read and write to include two parts, one is used to store parameters Number dug chip comes with flash, the other part is the size of the external expansion of 8M Flash M25P64, which is mainly used to store image data. STM32F1 Ox has two built-in flash memory programming. Online programming (In-Circuit Programming-ICP) mode is used to update the entire contents of the flash memory and it downloads the user application via JTAG or SWD protocol to the microcontroller. ICP is a fast and efficient process, eliminating packaging and stem problems. Way correspond with ICP, made in the program (In-Application Programming-IAP) microcontroller can be used to support any one of the communication interfaces (such as I / O ports, USB, CAN, UART, etc.) to download a program or data memory. IAP allows runtime reprogram the flash memory contents. However, IAP requires at least part of the program has been burned by ICP a flash block.

Control center receives data using the interrupt mode, interrupt-benefit is the ability to save system resources and improve the response speed and running speed of the system. Note needs, the master node is in the center of the beginning of time to disconnect them, and upon receiving the interrupt signal, after receiving the data, temporarily masked interrupts, and other data-all 
processing is complete before turning on interrupts, which It is to increase the speed of the system, no need to enter each interruption, especially when the received image data, if every word is bound to break into the waste of time. As shown, the program designed a counter, it is because in a wireless communication, there may be external factors or their own, leading to loss or interruption of communication, so the design of a counter, so that the main receiving point in the past a certain time, if no data is received or did not receive end flag pictures are automatically out of the receiving program returns to the main go.

When the invasion, or theft, the need for image acquisition and saves the image data into the system, providing strong evidence for the future fight against crime, the design of the image data through the SPI bus is stored in an external FLASH, the design on the outside flash M25P64 in a maximum of 20 images stored data when stored in 21 pictures, covering the first image data, and so on, for the need to design a counter value, to record the number of pictures stored in this system, referred to as photo-num, each received a full image data, photo_num from Canada once; information to be stored in addition to the image data itself, but also the length of the image data and the image shooting time, both types of data are needed storage. M25P64 is a 8M size of Flash, a total of 128 sectors, each sector can store 65,536 bytes of content, and tested, each picture data is generally not more than 7000 bytes, so with one sector to save picture data is sufficient.

\section{Conclusion}

Compared with traditional networks, wireless sensor network is a data-centric self-organizing wireless network and it has a quick temporary network, network topology changes dynamically, strong anti-destructive, no wiring, and data acquisition, transmission, processing and other advantages. It can be widely used in intelligent monitoring system design in order to improve people's living standards.

\section{References}

[1] Huifang Zhou: IEEE Communications Magazine, Vol. 6 (2014) No 53, p.25-26

[2] Hongli Zhang: Analytical and Chemistry, Vol. 12 (2015) No 27, p.74-76

[3] Qin Guo: China New Technology Products, Vol. 1 (2016) No 33, p.11-14

[4] Jieming Liu: Communications Magazine, Vol. 3 (2012) No33, p.121-124

[5] Can Liu: Bioinformatics and Biomedical Engineering, Vol. 3 (2011) No33, p.121-124 